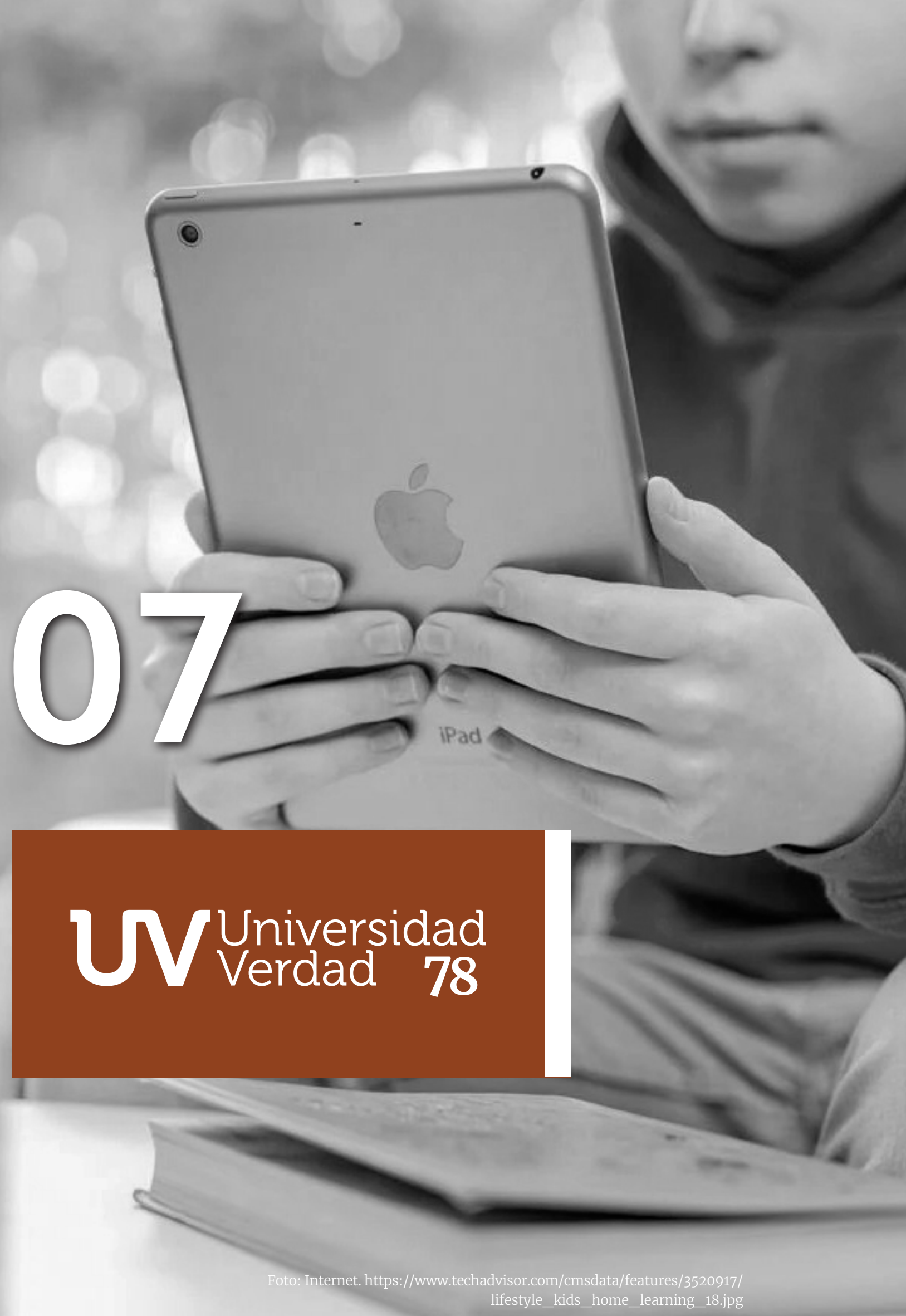




\title{
LA CONSTRUCCIÓN DEL CONOCIMIENTO DE LA EDUCACIÓN INCLUSIVA'
}

\section{The construction of knowledge of Inclusive Education}

\begin{abstract}
iD Aldo Ocampo González. Centro de Estudios Latinoamericanos de Educación Inclusiva (CELEI), (Chile) (aldo.ocampo@celei.cl) (https://orcid.org/0000-0002-6654-8269)
\end{abstract}

\section{Resumen}

Este artículo explora los mecanismos de construcción del conocimiento de la educación inclusiva. La inclusión no se limita a ningún campo en específico, no así la educación inclusiva. Lo inclusivo en el mundo contemporáneo desborda los significantes canónicos sobre los que se erige la función edipizante del calificativo a la fuerza subordinante, dominadora y normativa de lo especial. Nada comparte en su base genealógica, función, alcance y naturaleza la educación inclusiva con la educación especial. Si este error de proximidad objetual ha sido establecido, se debe, en parte, a la incapacidad de comprender la diversidad de objetos que en ella participan, así como, las debilidades de comprensión del término. El trabajo analiza las designaciones que impone su naturaleza epistemológica 'post-disciplinar', traza un signo supra-disciplinar y una fuerza generadora de orden diaspórica y nomadista, mecanismos sustentados en lo no-lineal -manifestación incardinada en lo rizomático-, la des-familiarización y producción de otros recursos heurísticos. El trabajo concluye que la educación inclusiva construye una epistemología neo-materialista, una afirmación crítica con el presente.

\section{Abstract}

This article explores the mechanisms of construction of knowledge of inclusive education. Inclusion is not limited to any specific field, not inclusive education. The inclusive in the contemporary world overflows the canonical signifiers on which the oedipal function of the qualifier is erected to the subordinating, dominating and normative force of the special. Nothing shares in its genealogical, function, scope and nature inclusive education with special education, if this error of object proximity has been established, it is due, in part, to the inability to understand the diversity of objects that participate in it, thus as, the understanding weaknesses of the term. The work analyzes the designations imposed by its epistemological 'post-disciplinary' nature, traces a supra-disciplinary sign and a generating force of diasporic and nomadic order, mechanisms supported by the non-linear -manifestation incardinated in the rhizomatic-, de-familiarization and production of other heuristic resources. The work concludes that inclusive education builds a neo-materialist epistemology, a critical statement with the present.

\section{Palabras clave}

Epistemología de la educación inclusiva, transposiciones heurísticas, nomadismo y diasporismo, neo-materialismo analítico, diagrama contingente de relaciones.

\section{Keywords}

Epistemology of inclusive education, heuristic transpositions, nomadism and diasporism, analytical neo-materialism, contingent diagram of relationships.

1. Este artículo corresponde a la conferencia leída por invitación en el Ciclo de Conferencias: "Resignificando las diferencias", organizado por la Facultad de Filosofía, Letras y Ciencias de la Educación de la Universidad del Azuay, Ecuador, el día 16 de diciembre de 2020. 
1.

\section{Introducción. La construcción del co- nocimiento de la educación inclusiva impone una concepción epistemológi- ca neo-materialista}

La epistemología de la educación inclusiva creada por Ocampo (2017) sostiene su actividad cognitiva en lo micropolítico, lo diaspórico, el nomadismo, la traducción, las topologías heurísticas y la performatividad de lo re-articulatorio, develando una figura de naturaleza post-disciplinaria, porosa y un centro heurístico de carácter relacional. No obstante, sus fuerzas definitorias asumen algunas características epistemológicas de carácter neo-materialista, es una forma de escape a los sistemas logo-céntricos de producción del saber.

La educación inclusiva al constituir una epistemología de la dispersión y del movimiento, conduce incesantemente a establecer otros modos de relación con la gran diversalidad de recursos constructivos que confluyen y circulan en su centro heurístico; en él, cada uno de los elementos se afectan unos a otros abriendo nuevos puntos de mutaciones y cambios creativos desconocidos que producen nuevos ángulos de visión. Las formas constructivas del conocimiento de la educación inclusiva operan a través múltiples capas y vectores multi-direccionados, inaugurando una actividad heurística mediante transposiciones desconocidas sostenidas en interconexiones híbridas y alterativas, generando nuevos objetos de estudio que no siempre, puntualizan sobre cuestiones educativas - actividad extra-disciplinar $^{2}-$. La educación inclusiva inaugura un nuevo campo de investigación. Tal como se encuentra concebida hoy -forma neo-especial- devela una insuficiente producción de conceptos y prácticas teórico-metodológicas. Su naturaleza epistemológica 'post-disciplinar' traza un signo supra-disciplinar y una fuerza generadora de orden diaspórica y nomadista, mecanismos sustentados en lo no-lineal -manifestación incardinada en lo rizomático-, la des-familiarización y producción de otros recursos heurísticos, todas ellas, atravesadas por complejas formas de rearticulación y traducción, estableciendo un tipo de ciencia que configura y se funda en la dispersión, en lo a-centrado y en la complejidad.

Al presentar una trama composicional de sistema abierto devela un terreno en perpetua transformación, un campo y objeto que es un proceso en proceso; un punto de encuentro entre trayectorias diversas en constante mutación. Esta es una de las características que definen de mejor manera el carácter neo-materialista de la naturaleza epistemológica de la educación inclusiva. La epistemología de la educación inclusiva planteada por Ocampo (2017) debe ser concebida como un salto de la imaginación heurístico-político orientada a cuestionar los términos ideológicos de la trama liberal que sustenta los argumentos sobre inclusión y educación inclusiva desde un efecto falsacionista o mainstream -refiero al efecto mimético de lo especial ${ }^{3}-$.

La complejidad y multidimensionalidad de los argumentos, formas enunciativas, recursos constructivos, campos de confluencias geografías intelectua-

2. Actividad heurística que trabaja conectando campos alejados en el ensamblaje de objetos de conocimientos, los que dialogan en un punto particular, no siempre reconocible.

3. Metáfora acuñada por Ocampo (2019 y 2020) quien explica cómo los planteamientos actualmente empleados para justificar la presencia de lo inclusivo reafirman un efecto de imitación de la montura epistémica y didáctica de la educación especial, asumiendo una condición de disfraz heurístico. Para más información, véase el artículo que lleva este nombre. 
les, herramientas metodológicas y conceptuales, exigen la co-presencialidad de un examen topológico no solo referido a los grados de vinculación y posición entre cada uno de sus recursos constructivos, que a su vez, son topologías complejas en la producción de su saber auténtico, exige adentrarnos en su malla de relacionalidad multidireccionada. Si su práctica cognitiva se centra en el diasporismo y en el nomadismo, entonces, su sentido de producción del conocimiento reafirma un compromiso con la creación de nuevos saberes y conceptos -objetivo heurístico que constituye una de sus principales tareas-, asume una forma epistemológica en permanente devenir, característica que constituye el motor principal del trabajo post-disciplinar, redefiniendo la relación entre cada uno de sus campos y recursos constructivos. Su carácter orgánico asume que "el devenir virtual e intensivo reemplaza el principio imperante de semejanza, identidad, analogía y oposición. La continuidad entre el presente y actual, Chronos y Aion, activa múltiples líneas genealógicas de resonancia (Deleuze, 1988)" (Braidotti, 2018, p.18).

Las prácticas de producción del conocimiento de lo inclusivo trabajan en múltiples ritmos de aceleración y velocidades articulando su tarea por fuera de la lógica neoliberal. Es una operación de movilización de un comercio de zonas y puntos de contacto, intereses, preocupaciones, recursos constructivos, proyectos políticos, proyectos de conocimiento en resistencia, compromisos éticos, dislocaciones ontológicas, movimientos sociales e instrumentos conceptuales; en suma, un territorio multifacético. La inclusión como campo y fenómeno y la educación inclusiva como circunscripción intelectual, ambas, cada una a su manera, concebidas como dispositivos heurísticos exigen un reajuste de las leyes científicas que regulan los modos comprehensivos del quehacer epistemológico en el mundo actual. Sin duda, la relación heurístico-política de sus territorios exige concebir su campo de fenómenos en términos de singularidad compleja, ensamblaje afectivo y entidad vitalista relacional, develando un profundo cambio en los mecanismos de proliferación de sus objetos, contornos metodológicos y herramientas constructivas requeridas, las que, sin duda, son imprevisibles. Su red epistemológica adopta lo que Braidotti (2018) denomina "singularidad situada y compleja de los sujetos de conocimiento contemporáneos" (p.19). La inclusión nunca satura los procesos del devenir, razón por la que se encuentra en estado de redoblamiento, apertura ambivalente y movimiento rizomático, cuyas territorialidades heurísticas acontecen por medio de un conjunto de múltiples capas, inaugurando una matriz constructiva que impone un proceso dinámico y estratégico. Es un sistema de recomposición o recognición del conocimiento educativo contemporáneo. La educación inclusiva forja un ensamblaje afirmativo comprometido con el estudio y comprensión del presente, una forma edificante y creativa para problematizar y pensar ante la multiplicidad de problemas educativos.

¿Dinámica no-lineal? Sin duda, la comprensión de la naturaleza epistemológica de la educación inclusiva no puede entenderse a partir de recursos constructivos, proyectos de conocimientos y geografías intelectuales de manera aislada, o bien, bajo una figuración paratáctica, sino, puntualiza en las interfaces, los mecanismos de comunicación por rearticulación y traducción de estos. La naturaleza no-lineal del campo devela un complejo terreno de movimientos estratificados que construyen estructuras heurísticas afectadas por singulares mecanismos de territorialización y desterritorialización atravesados por un ensamblaje de elementos heterogéneos. Si el campo de la educación inclusiva alberga en su interioridad una gran constelación de recursos analíticos que se abren a la contingencia para dar pauta a la creación de otro ensamblaje (De Landa, 2016). Su construcción heurística asume y reafirma una concepción radical de lo múltiple, pero ¿qué es lo que caracteriza su particularidad en términos constructivos? Fundamentalmente, en términos deleuzianos asume un carácter no-predicativo de lo predicable, pues su morfología no es del todo dueña de sí misma, establecida y determinable como otros campos del conocimiento. Esto acarrea un corpus de tensiones de orden heurístico. La escena de la multiplicidad como forma constructiva construye lo múltiple al mismo tiempo que lo describe, un sistema de performatividad, rearticulación y apertura por redoblamiento de cada uno de sus elementos.

Los signos que mejor describen la epistemología de la educación inclusiva son los de sistema diaspórico, heterológicos y heterogenésicos, formas que, a su vez, constituyen parte sustantiva de sus principios epistemológicos; todos ellos, a su manera, develan un sistema heterogéneo de lo heterogéneo, una ló- 
gica constructiva fundada en las operatorias de la multiplicidad. En términos deleuzianos construye otra potencia intelectual. En ella, cada singularidad constructiva impone una imagen de pensamiento abierto cuya conectividad solo se cierra para abrirse hacia rumbos desconocidos. Lo abierto implica lo indeterminado, en palabras de Mengue (2008):

[...] el problema es cómo lo implica. Sin duda, no contra la posibilidad de cualquier determinación y tampoco contra la existencia de un algo determinado. Nada más ajeno a Deleuze que el vano compromiso con la infinitización de lo finito. Dicho brevemente, si en el pensamiento deleuziano lo abierto implica lo indeterminado es sólo como respuesta a la pregunta por el emplazamiento que permite hacer posible el pensamiento de una determinación cualquiera o de cualquier determinado en condiciones de estricta inmanencia. Lo abierto implica lo indeterminado. Lo indeterminado es el caos. Sí, pero el caos no es lo opuesto de cualquier determinación sino aquella instancia que, en tanto encarna una potencia absoluta de diferenciación tiende a hacer posible -aunque no necesariamente composible- la existencia de algo tal como un determinado. Remitidos entonces a la cuestión de la determinación, lo central del aporte deleuziano no reside en su supuesta opción por lo indeterminado sino en su reformulación de las condiciones bajo las cuales éste se relaciona con la determinación y lo determinado: lo indeterminado no es lo contrario de lo determinado sino su condición, aquel ámbito lógico u ontológico que hace posible cualquier determinación y que permite a los determinados existir unos junto a otros aun cuando resulten incapaces de componerse. (p.13)

El campo epistemológico de la educación inclusiva surge a través de líneas de pensamiento heterogéneas, es un territorio del pluralismo presente en cada uno de sus elementos constructivos "que piensa las cosas como multiplicidades, es él mismo múltiple, constituye él mismo, y en el plano del pensamiento, lo múltiple. Esta lógica de lo múltiple, en tanto que existe, existe ella misma, como todo lo que existe; a saber, como de lo múltiple" (Mengue, 2008, p.44). Esta compresión epistemológica de lo inclusivo encuentra diversas clases de paralelismos con lo que Mengue (2008) denomina
[...] una teoría de las multiplicidades - de la diferencia y de su repetición (=DR), de los acontecimientos (=LS), de los agenciamientos maquínicos y de los flujos de deseos (=Aai), de los rizomas y de conjuntos de líneas que forman mesetas (=MP). Así, nuestro problema del comentario está legítimamente resuello: si hay una teoría posible de lo múltiple, habrá también la posibilidad de dar cuenta de la teoría (deleuziana) de lo múltiple, aunque a su vez ella sea múltiple. (p.45)

2.

\section{Los conceptos de la educación inclusiva nacen de la multiplicidad}

El estudio crítico-epistemológico de las categorías que ensamblan la comprensión de la fuerza teórica, metodológica y política de la educación inclusiva auténtica, coherente con sus demandas heurísticas de post-disciplinariedad y con sus exigencias ontológicas fundadas en un diagrama de posiciones contingentes, transitivas y relaciones para albergar a la multiplicidad de singularidades, supone un reconocimiento crucial de carácter alterativo: los conceptos que fundan el aparato epistemológico y político de la educación inclusiva, por consiguiente, pedagógico; no tienen relación alguna con la matriz de regulación esencialista-individualista montada sobre las operaciones de saber-poder producidas por los marcos de inteligibilidad del humanismo clásico, cuyos instrumentos conceptuales trabajan a favor de regulaciones onto-cognitivas de la abyección. No hay que olvidar que los conceptos no solo desempeñan una función de regulación de las condiciones lexicales de todo dominio -tarea más visible-, sino que, contribuyen a comprender o distorsionar las coordenadas de trabajo de su objeto. En campos emergentes como el aquí analizado, los conceptos constituyen un punto de partida para responder a la ausencia de métodos, tal como propone Bal (2009) los conceptos pueden reemplazar la actividad de los métodos en territorios donde el analista -investigador- no dispone de muchos medios en los que apoyarse.

Si lo conceptos son herramientas que facilitan el diálogo y la interacción entre diversos recursos episte- 
mológicos y regionalizaciones que deambulan de un lado a otro sin un rumbo fijo, entonces, en su dimensión analítica, constituyen "representaciones abstractas de un objeto" (Bal, 2009, p.35), fomentando un conjunto de herramientas para el análisis de objetos de diversa naturaleza como son los que confluyen y albergan el terreno en discusión.

¿Qué es lo que hacen los conceptos de la educación inclusiva? Antes de responder a esta interrogante, es necesario aclarar que sus instrumentos conceptuales necesitan ser comprendidos y empleados por todas las personas, incluso profesionales en campos alejados de su actividad, pues, su materialidad afecta alterativamente a diversos campos del desarrollo humano; cada uno a su manera, constituyen mecanismos de regulación de los objetos devenidos en prácticas otras de imaginación e interpretación. Otro punto espinoso asume que no todos los conceptos que ensamblan la figuración epistémico-discursivo mainstream de lo inclusivo pueden ser significados con esta denominación, pues carecen de estatus metodológicos. Un buen ejemplo de ello, es el sintagma 'necesidades educativas especiales'. Solo confirman un efecto de subjetivemas y palabras-comunes que carecen de unidades de significado por giro rearticulador con las demandas de su gran aparato cognitivo. Bal (2009) agrega que, ningún concepto está ajeno a condiciones de ambigüedad producto de su marcado carácter en movimiento. La pregunta que preside a este cúmulo de respuestas bordea la necesidad de abordar con precisión los esquemas teóricos de sus conceptos fundantes y sus patrones de interactividad. Cada uno de estos viaja por regionalizaciones y geografías intelectuales y comunidades de adherencias dispersas.

Si reconocemos que los conceptos de la educación inclusiva auténtica surgen del centro crítico de la multiplicidad, lo que desestabiliza la pureza y sujeción del esencialismo y su compromiso con el humanismo clásico, en cuanto a sus modos de comprender la realidad y las posibilidades del sujeto. La multiplicidad en tanto noción analítico-política es atravesada por diversos fenómenos relacionales ligados al género, al antirracismo, la justicia, a la interculturalidad, a la subalternidad, etc.; buena parte de ellos denotan la creación de proyectos políticos y de epistemologías radicales. Otra advertencia reconoce que la multiplicidad se convierte en un con- tenedor genealógico de las grandes nociones sobre las que se erigen los entendimientos de lo inclusivo Entre ellas encontramos: a) heterogeneidad, b) alteridad, c) diversidad, d) diferencia, e) singularidad, f) otredad, entre muchas otras. Otra dimensión asume que la multiplicidad es un concepto a través de la cual se construye un diagrama de posiciones contingentes para abordar los problemas de carácter oblicuo y transitivo de diversas colectividades de personas. Las diferentes preocupaciones que enlaza la noción de multiplicidad en este terreno, se han sostenido en debates procedentes del "feminismo, análisis de clase, antirracismo, políticas de gays y lesbianas, psicoanálisis, postestructuralismo y demás" (Brah, 2011, p.38). Finalmente, los conceptos que surgen del centro de la multiplicidad trabajan junto y son inmanentes, permitiendo entender diversas clases de procesos y análisis históricos, culturales y sociales que marcan los signos del presente. La heterogeneidad, la alteridad, la diversidad, la diferencia, la singularidad y la otredad trabajan inextricablemente unidos, definiendo nuevas relaciones contingentes y modos de relacionamientos que toman diferentes formas de acuerdo con sus contextos de práctica.

Si bien, las nociones que surgen del centro de la multiplicidad adoptan un estatus abstracto, sin embargo, agrega Brah (2011) que, "tienen efectos muy «reales», aunque de diverso tipo, en diferentes grupos" (p.114). Esto permite puntualizar en las prácticas y experiencias subjetivas de sus usuarios, los que "emergen como conceptos relacionales constituidos en y a través de la experiencia «interior» y «exterior»" (Brah, 2011, p.150) a cada una de tales colectividades. Al constituir la educación inclusiva un campo de agenciamientos conceptuales de carácter relacional exige pensar su campo de problemas a través de la experiencia, la subjetividad, la identidad y la relación social, tal como señala Brah (2011). 
3.

\section{La naturaleza post-disciplinar de la educación inclusiva: ¿qué es, cómo opera e investiga?}

La pregunta por la base epistemológica de la educación inclusiva constituye un ámbito analítico y de trabajo invisibilizado por su comunidad de práctica, encontrar una respuesta acertada fomenta la apertura de nuevos ángulos de visión y conocimientos que han permanecido ocultos entre diversos recursos analítico-metodológicos; especialmente, cuando sus contornos definitorios quedan atravesados por objetos teóricos que no comparten ninguna hebra común, ni mucho menos un lenguaje, develando una compleja naturaleza diaspórica y peculiares trayectorias rizomáticas. Los territorios de la educación inclusiva reafirman un complejo campo de mutaciones, alteraciones y transformaciones sistemáticas. La pregunta por su base epistemológica sugiere una advertencia preliminar: no es una tarea sencilla delimitar los ejes de configuración heurísticos de un campo en permanente movimiento debido a que sus ensamblajes acontecen a través de diversas convergencias epistemológicas que son aglutinadas en un complejo post-disciplinar. Lo 'post-' actúa en términos de un dispositivo de ruptura, giro y alteratividad de cada uno de sus recursos constructivos, la comprensión de sus fenómenos se enuncia y escucha más allá de múltiples proyectos de conocimiento que confluyen en su gran campo de fenómenos. La vida del campo surge mediante la combinación rearticuladora y el giro de diversas clases de materiales, imponiendo el signo de una construcción experimental del saber en la que cada uno de ellos participa activamente.

La política general de construcción del conocimiento de la educación inclusiva necesita disponer de un conjunto de herramientas para responder a múltiples desafíos, asumiendo problemas multifacéticos y complejos, lo que implica diversas redes y relaciones por redoblamiento entre diversas singularidades constructivas que animan a cada uno de estos a la transgresión, fomentando posibles desafíos y beneficios de epistemologías fundamentalmente diferentes que coexisten entre sus niveles de construcción. "Palabras como interdisciplinario, multidisciplinario o incluso no disciplinario han sido dis- cutidas desde diferentes puntos de vista en el campo creativo y las humanidades" (Niinimäk et al., 2018, p. 238). La pregunta por la base epistemológica de la educación inclusiva puntualiza en sus modos de interacción entre sus diversas convergencias heurísticas: lo multi-disciplinar, lo inter-disciplinar, lo trans-disciplinar, lo extra-disciplinar para llegar a la configuración de una figura de orden post-disciplinar; las líneas de fuga son las que promueven su fuerza heurística. Examinemos, aunque brevemente, cada una de estas manifestaciones que se convierten en un dispositivo, en un lugar y en una estrategia que habilita un cambio de lógica-de-sentido, posibilita la emergencia de un corpus de tensiones sin reducir arbitrariamente sus implicaciones analíticas, sugiere un cambio de lógica en la forma de producir su conocimiento, legitimar sus modos de aproximación y comprensión de su objeto. En suma, es un cambio de lógica analítica, semántica y metodológica con grados de afectación creciente. Un punto de fuga sugiere la renuncia a la armonía trazada por el signo de lo diverso que inspira el eclecticismo en tanto tecnología del positivismo, introducido por vía de la reunión de elementos de diversa naturaleza. Un punto de fuga es un punto de inflexión, una totalidad reconstruida, su fuerza dislocadora y alterativa produce un cambio que inaugura otros caminos posibles para experimentar y pensar las configuraciones heurísticas de los territorios de lo inclusivo.

Ninguna de las definiciones que abordaremos a continuación disfruta de un estatus consolidado ni de una fuerza analítico-metodológica del todo clara. Frecuentemente, podemos encontrar diversas acepciones, posiciones y discusiones al respecto, lo que devela una amplitud de los términos y una política de todo vale. Ciertamente, las formas no-disciplinares del conocimiento asumen en su superficialidad constructiva un efecto paratáctico y eclecticista; ambos a su forma, develan la mezcla desmedida de diversos recursos metodológicos, políticos, discursivos, analíticos conceptuales, entre otros, deviniendo en una praxis cognitiva de carácter parasitaria. El eclecticismo es erróneamente concebido como una forma holística de producción del conocimiento, que encubre la fuerza expresiva del positivismo. Por su parte, la acción paratáctica devela que ciertas formas de producción del conocimiento que desbordan el funcionamiento disciplinar, tienden a trabajar en la superficialidad de la simple reunión de elementos diferentes sin entrelazar sus puntos de inter-refe- 
renciación y relación entre ellos. Es muy frecuente observar que, en campos como estos, se cruzan fronteras que no se transforman. ¿Qué relación tienen estos argumentos con la pregunta por la base epistemológica de la educación inclusiva? Su objeto y campo de fenómenos no puede ser delimitado en los marcos y paradigmas de ninguna disciplina, producto que estos desbordan la habitualidad de sus formas comprehensivas. Tampoco pertenece con exactitud a la regionalización de la educación especial y a otros dominios que confluyen en la arquitectura su ensamblaje heurístico-político.

La educación inclusiva como circunscripción intelectual devela un campo en permanente movimiento, que opera por deslimitación y configura una singular y desconocida red de agenciamientos analítico-metodológicos. Si la educación inclusiva ha sido vinculada con la educación especial -principal fracaso cognitivo y fallo genealógico- se debe, en cierta medida, a la incapacidad de esta para abordar y comprender su gran abanico de objetos visuales, analíticos, metodológicos, éticos, políticos, etc. Enfrenta, además, un problema en la determinación de sus condiciones de identificación, descripción y posicionamiento de sus objetos ${ }^{4}$. La travestización del calificativo y su fuerza con lo especial se debe a la incapacidad de los investigadores para aproximarse al propio término. Tomar la 'educación inclusiva' como 'educación especial' es condenarla al mismo fallo. Mientras que la segunda, es una forma de inclusión, un objeto específico dentro de su gran abanico de tópicos y fenómenos de análisis; en cambio, la inclusión es una forma mucho más compleja, en la que se cruzan, desbordan y rearticulan una multiplicidad de elementos que dan vida a su campo de producción, reconociendo que esta no es una forma de lo especial, es algo mucho más grande y abarcador. Si bien, la educación especial no puede ser ignorada -palimpsesto epistémico- tampoco puede ser entendida bajo un campo de relaciones equívo$\cos$ y/o restrictivas. No necesita incorporarse a otras disciplinas, sino traducir y crear nuevas aleaciones a partir de sus aportes derivativos que emergen espontáneamente en su viaje por una inmensidad de territorios heurísticos. Sin duda, la falta de claridad sobre la naturaleza de dicho dominio sigue siendo el punto más crítico de su teoría.
Si bien la educación inclusiva desarrolla un objeto post-disciplinar, un campo de fenómenos que escucha y enuncia sus fenómenos más allá de múltiples clases de problemas, discursos, conceptos, teorías, metodologías, territorios, proyectos políticos, proyectos de conocimiento y compromisos éticos, entre otros; configurando un territorio atravesado por singulares agenciamientos heurísticos, que a pesar de inscribir su actividad más allá de cada uno de estos recursos articula cuestiones específicas sobre objetos específicos. Es un campo de aperturas, saltos, traducciones y redoblamientos permanentes. La convergencia multidisciplinaria que participa del ensamblaje del campo se torna visible mediante el encuentro, diálogo y participación de diversas geografías intelectuales que aprovechan determinados aportes disciplinarios devenidos en un ensamblaje superficial y un encuentro de baja intensidad. Hasta aquí, sus aportes no se imbrican en la centralidad de los territorios, sino que, residen en planos parcializados y alejados en sus contornos definitorios. El carácter flotativo de cada uno de sus recursos es lo que posibilita el movimiento; es él el que habilita la apertura y el redoblamiento hacia una convergencia inter-disciplinaria en la que arriban diversos métodos, conceptos y conocimientos de diferentes regionalizaciones epistémicas, incluso, se observa la presencia de diversos proyectos políticos, como el feminismo, el antirracismo, etc. El flujo de transferencia de diversas clases de recursos constructivos habilita la pregunta por los mecanismos de comprensión, legibilidad, posicionamiento, relación con y traducción de cada uno de sus recursos, exigiendo ir más allá de una síntesis de cada uno de sus enfoques convergentes. Develar cada una de estas formas de síntesis, constituye un punto nodal crítico para develar la naturaleza de los ensamblajes por rearticulación. La convergencia inter-disciplinaria en el terreno de lo inclusivo según Biagioli (2009) actúa a través de

[...] ciclos de retroalimentación, cuando las perso-
nas recuperan los conocimientos de otras discipli-
nas en su investigación disciplinaria individual, o
cuando trabajan juntos en un proyecto que se su-
perpone a múltiples disciplinas. Cuando ese tra-
bajo cruza el límite difuso entre superposición y
mallado, terminas con nuevas disciplinas. (p.819)

4. Esta razón justifica la necesidad heurística de Ocampo (2016) de construir mecanismos de examinación topológicas para develar las coordenadas de relación y proximidad entre ambas figuraciones analíticas. 
Biagioli (2009) insiste, agregando que,

[...] la interdisciplinariedad no es una familia de disciplinas, ni una disciplina más completa y rica, ni un nuevo brote o rama del árbol del conocimiento. Es una colaboración de problemas específicos que tiene lugar dentro de una ventana temporal limitada y en lugares que pueden tener poco que ver con los departamentos e institutos estándar. Se espera que estas colaboraciones interdisciplinarias produzcan resultados importantes, pero no necesariamente tengan una vida institucional duradera. (p.822)

La convergencia trans-disciplinar es clave, al igual que la inter-, producto que fabrica entendimientos que operan 'a través de', 'al otro lado de' y 'detrás de' cada uno de los marcos disciplinarios, territorios y regionalizaciones convergentes en la rostricidad de lo inclusivo. Lo trans-disciplinar se encuentra estrechamente vinculado al concepto de emergencia introducido por vía de los sistemas complejos. La trans-disciplina comparte con las post-disciplinas la tarea por la producción de lo nuevo, especialmente, de un nuevo objeto y campo, dos tareas espinosas en los estudios sobre educación inclusiva. El problema no se reduce exclusivamente a ello, sino que, reside en la síntesis que cada dimensión elabora. Nos enfrentamos a convergencias que son móviles, plásticas y mutantes, es esto, lo que reafirma y define uno de sus principios epistemológicos más importantes: la heterogénesis. Esta contribuye a visualizar un mapa de múltiples direcciones en la construcción de sus territorios y constituye una tarea inseparable de la pregunta acerca de cómo interpretar la trayectoria de cada recurso y convergencia heurística, los que se penetran, dislocan, contagian, contaminan, traducen, redoblan y rearticulan permanentemente. La heterogénesis inaugura un campo de potencialidades que aparecen y desaparecen, describe las fisuras y grietas que emergen de las fuerzas destituyentes que intentan "conseguir una acumulación de fuerzas provisionales mediante el reconocimiento de unos objetivos comunes mínimos" (Martínez, 2020, p.s/r.) inaugurando un nuevo plano o diagrama de relaciones -lo constituyente-, lo que entraña un profundo movimiento de descodificación y desterritorialización de cada uno de sus recursos constructivos. La noción de diagrama de relaciones constituye otro concepto epistemológico central en la exploración de las convergencias heurísticas de lo inclusivo. Sus usos entrañan las fuerzas de lo invisible que produce efectos visibles en la realidad, así como, las tecnologías de territorialidad, desterritorialización y re-territorialización. El diagrama heurístico de la educación inclusiva se encuentra regulado con diversas formas de enredos, cuyos efectos pueden ser leídos en términos de

[...] diagrama se concibe como una máquina autopoiética que no solo le otorga una consistencia funcional y material, sino que le exige desplegar sus diversos registros de alteridad, liberándolo de una identidad encerrada en simples relaciones estructurales" (Guattari, 1995a, pág.44). "Liberar" aquí se aplica a escapar de un "orden esquemático" predeterminado impuesto a la máquina, que funciona en el horizonte de la muerte y la destructibilidad. El uso que hace Guattari de Diagrama se opone a la idea de la reproducibilidad misma de una máquina técnica (como en la computadora y sus partes). (Panayotov, 2016, p.s/r.)

Si bien la educación inclusiva surge de múltiples enredos genealógicos devela una singular trayectoria rizomática cuyos desarrollos no se encuentran unificados ni por un objeto común ni por un lenguaje teórico único, sino múltiples recursos y no fácilmente reconocibles. Al romper con las dicotomías, las normatividades y los sistemas analíticos de fijeza del actuar canónico, se produce una gran diversidad de nuevas interfaces y conexiones entre cada uno de los elementos que integran sus ensamblajes cuyo sentido de apertura no cae en el juego de legitimar un sistema de elasticidad que permita la confluencia y juntura de todo con todo, empresa que resulta estéril y es introducida por un cierto efecto de ceguera ante los efectos y modos de proximidad a los diversos niveles heurísticos del campo.

El carácter rizomático de los territorios de la educación inclusiva devela una construcción multifacética, multinivel, multi-escalar y multi-versal. No nos enfrenta a un efecto de crisis disciplinaria, sino a la producción de otros modos de conocimientos que habitan el campo de lo raro, lo no-visto, lo no-pensado e imaginado. La presencia de diversas convergencias heurísticas en la estructuración del campo de conocimiento de la educación inclusiva 
no termina aquí, más bien, identifica la presencia de múltiples hilos que forman una coyuntura cognitiva acentrada - conectada sin ninguna unidad centralque opera en proximidad al concepto de artesanía propuesto por Sousa (2009). Cada una de las convergencias heurísticas antes señaladas, se componen de dimensiones y direcciones en movimiento, cada una de estas operan por variación, expansión, conquista, captura, ramificaciones, cuya multicomplejidad devela múltiples entradas, las que resultan parciales, pues, en algún punto necesitan de traducción de sus unidades de constitución y significación de acuerdo con las manifestaciones de lo inclusivo. La presencia de diversas convergencias epistemológicas es propia de los campos alécticos o de la pluralidad epistemológica, regulados por los principios de conexión, heterogeneidad, multiplicidad y ruptura asignificante, adoptando la figuración de un ensamblaje colectivo, un territorio trasrelacional que se encuentra en permanente devenir, adaptación, modificación y transformación, todas ellas, atravesadas por el signo de lo alterativo.

Las formas de producción del conocimiento de la educación inclusiva explicitan una gran variedad de mecanismos creativos que no solo refuerzan un conjunto de capacidades críticas de carácter afirmativo para leer el presente y signos problemáticos y evanescentes, sino también, orienta su campo de inquietudes hacia la construcción de un proyecto de conocimiento en resistencia multidireccional y multicapa, interesado en develar las condiciones heurístico-políticas que fomentan la emergencia de su campo de autenticidad. Para ello, analiza las formaciones de producción de su conocimiento entendidas como condiciones político-culturales, articuladas sobre relaciones de poder-, su práctica académica en la Ciencia Educativa y sus implicaciones para la construcción de la subjetividad. Implicaciones que claman un análisis pormenorizado acerca de sus lecturas y usos específicos de su aparato conceptual, ontológico, metodológico y heurístico. La educación inclusiva es el acto de pensar sobre el futuro, la inclusión como aparato analítico-metodologico devela una naturaleza compleja y heterogénea cuya existencia de múltiples cosas impone un sistema de simultaneidad de trayectorias, son cosas en desarrollo al mismo tiempo. Todo ello, produce un cambio del terreno analítico-metodológico.
La pregunta por la base epistemológica de la educación inclusiva es una cita con tres ámbitos definitorios cruciales: a) los elementos de delimitación del sujeto conocedor en este marco, b) sus niveles de cientificidad y credibilidad y c) su trama de responsabilidad ética frente a los problemas apremiantes del mundo que habitamos. La educación inclusiva es un campo emergente que surge de diversos enredos genealógicos, conflictos entre sus geografías intelectuales y proyectos de conocimientos, mediaciones y diálogos no siempre armónicos, devela un territorio que fluye a través de trayectorias rizomáticas que son rearticuladas para abrir nuevos ángulos de visión. Como campo emergente surge de la confluencia del feminismo, el antirracismo, lo de-/post-colonial, el anti-humanismo, el post-humanismo, la filosofía de la diferencia, la interculturalidad crítica, la interseccionalidad, la filosofía analítica, la filosofía política, los nuevos estudios de democracia, los estudios de género, los estudios queer, los estudios culturales, la teoría crítica, entre otros; cada uno a su manera , otorgan herramientas para pensar e intervenir sobre cada uno de sus problemas más allá de sus aportes y críticas a una gran diversidad de problemas endémicos a los sistemas-mundo. Otra tarea crítica que enfrenta la educación inclusiva consiste en documentar como cada uno de estos proyectos políticos y de conocimiento, así como dominios disciplinares, inter- y tras-disciplinares fueron confluyendo en su gran ensamblaje heurístico. Nos enfrentamos aquí a la comprensión de la materialidad del fenómeno y a la pregunta acerca de cómo funciona por sobre qué es -interrogante de carácter metafísico-.

El problema que enfrentan los territorios de la educación inclusiva, a la luz de la pregunta por el corpus de elementos que definen su estatus de paradigma, alude a un conjunto de limitaciones descriptivas para llegar a la naturaleza real y profunda de las prácticas científicas que participan en su organicidad. En superación de ello es que concibo los contornos y las diversas formas delimitativas del campo en términos de ensamblajes y sistemas experimentales de producción, concepciones coherentes con dos nociones claves en las formas constructivas del conocimiento de lo inclusivo. El problema de la visión paradigmática devela "simplemente una construcción demasiado holística y pone demasiado énfasis en la cohesión intelectual y social de un grupo y en la uniformidad de su entrenamiento para que coincida con los escenarios notablemente diversos y cambiantes de la in- 
vestigación contemporánea" (Biagioli, 2009, p.819). Lo post-disciplinar es altamente fértil en la captura de ámbitos de producción del conocimiento, cuyos practicantes se organizan en torno a problemas, no disciplinas -caso singular por deslimitación de la educación inclusiva-, desafiando la organización de los espacios académicos y sus clásicas convenciones taxonómicas. En las disciplinas los objetos expresan con mayor fuerza un halo de estabilización, en las post-disciplinas, no, producto que surge del movimiento, la traducción y el pensamiento de la relación multiaxial; dando paso a nuevos objetos, nuevas categorías y prácticas para leer y abordar los signos del presente. Si bien la educación inclusiva evidencia una crisis en su identidad científica o un problema de comprensión de su índice de singularidad -problema común de todos los campos sintagmáticos como suelen ser denominados por Ocampo (2020)-, esta, en tanto territorio heurístico construye una política de identidad otra. El índice de singularidad permite definir a ciencia cierta qué es la educación inclusiva.

La educación inclusiva no es solo una gran constelación de recursos constructivos, sino un poderoso mecanismo de transformación del mundo conocido. Nos conduce hacia nuevos enfoques aún desconocidos en la educación, replantea nuestros modos de existencia, vivencia y relacionamientos. Nos invita a replantear el acto educativo, los modos de ejercicio de la maternidad, la paternidad, la hermandad, la forma de existir y pensar la vida. En suma, un profundo cambio alterativo de nuestra consciencia. Todo ello, define a la educación inclusiva como una práctica emergente y un sistema de emergencia temporal.

Tales aportes no solo contribuyen a ensamblar un concepto otro, que otorgue luminosidad a la turbiedad, a los contornos y a su índice de singularidad, sino que, reconstruye su red ontológica monista neo-spinozista imponiendo un compresión del ser en términos de pluralidad individual o singularidades múltiples creando nuevos estilos de relacionamientos, coordenadas de alteridad, formas de convivencialidad, modos de subjetivación y subjetividad; entiende al ser como una entidad encarnada - resultado del materialismo-, cuyas acciones teóricas brindan un marco afirmativo con el presente. La educación inclusiva crea un nuevo marco ontológico para comprender a los sujetos educativos, lo que exige examinar las políticas de ubicaciones de los investigadores y de los trabajadores de las políticas públicas en el interior de dicho campo de fenómenos, inscripciones que oscilan entre la materialidad y subjetividad de los espacios y la memoria histórica, que median sus interpretaciones. Sus formas interpretativas y mecanismos de producción marcan el rechazo del universalismo trascendental y la gramática dialectal de orden hegeliano, que articula su tarea sobre los clásicos sistemas categoriales de oposición binarista, y establece una nueva red de relaciones entre diversas formas de humanidad y de lo humano. El carácter creativo del campo es definido; en cierta medida, como un sistema que habita el doblete analítico de pensar-de-otro-modo sobre nuestra condición humana y las formas de producción del conocimiento del campo; propósito que para Braidotti (2018) consiste en "generar figuraciones o personajes conceptuales alterativos para el tipo de sujetos conocedores actualmente construido" (p.4) y sancionados por las exigencias del régimen auténtico de lo inclusivo.

A la luz de estas tensiones, la educación inclusiva construye un conocimiento desconocido, raro y no-imaginado, de carácter post-disciplinar, que surge de diversas convergencias heurísticas las que están produciendo una serie de posiciones alterativas sobre las formas de producción del conocimiento. Antes de avanzar, observo necesario efectuar una distinción, aunque parcial, crucial: la inclusión en tanto fenómeno excede el alcance de este trabajo, mientras que, la educación inclusiva como circunscripción intelectual y territorio heurístico constituye un ensamblaje post-disciplinar, un nuevo sistema cognitivo y de erudición, en rechazo de la simple integración de elementos de naturaleza heterogénea, sin lazos, anudamientos ni traducciones significativas para ensamblar otra forma comprehensiva sobe la imbricación de los fenómenos de los sistemas-mundo y los sistemas-educativos. Si bien, el campo de producción epistemológico de la educación inclusiva se compone de la presencia y convergencia de múltiples objetos de conocimientos, premisa que exige avanzar hacia el reconocimiento que esta trabaja con múltiples sujetos de conocimiento, rompiendo con el ideal normo-céntrico que universaliza las porciones del sujeto en el interior de su campo de fenómenos. Al definir la educación inclusiva desde la fuerza edipizante de lo especial, no le hace justicia, especialmente, porque los temas de análisis de este 
último calificativo, no irrumpen, ni dialogan con la creciente multiplicación de las desigualdades educativas, económicas y sociales, crean pseudo-efecto de trabajo, que impone una práctica que crea códigos de ordenación para proteger a diversos sujetos atravesados por estas clases de desigualdades, sin modificar e impactar en la vida de las personas. La educación especial es un marco de referencia insuficiente para enfrentar los dilemas de lo inclusivo en sus diversos niveles de trabajo.

La comprensión epistemológica de la educación inclusiva construye un diagrama y un predicamento multidireccional y multicapa, se interesa por subrayar sobre el corpus de elementos que entronizan su función en tanto fuerza en devenir. Una de sus tareas metodológicas consistirá en develar los objetos teóricos, empíricos, analíticos y discursivos que favorecen el intercambio protagónico y antagónico entre cada uno de sus recursos constructivos, dando cuenta de una naturaleza relacional, nómade y diaspórica - características centrales en la comprensión epistemológica de la educación inclusiva-. Cada una de estas implicaciones es altamente selectiva, funcionan en y a través de la multiplicidad de formas conectivas y rearticuladoras que denotan una heurística de referencias cruzadas que exigen de traducción y legibilidad al ensamblar un nuevo territorio. La educación inclusiva inscribe su función en tanto "relato teórico y políticamente informado del presente, que tiene como objetivo rastrear la producción de conocimiento y subjetividad (Braidotti, 1994, 2011a, 2011b) y exponer el poder tanto como atrapamiento (potestas) y como empoderamiento (potentia)" (Braidotti, 2018, p.3). La pregunta sigue siendo cómo abrazar los aspectos afirmativos de un nuevo conocimiento en la materia, para lo cual es fértil adoptar un marco de trabajo nómade, rizomático, viajero y diaspórico; leyes constructivas que legitiman nuevos vínculos e interfaces otras entre cada uno sus recursos constructivos confluyentes, develando un campo de múltiples e intensos intereses y e influencias, todas ellos, de carácter emergente. Este territorio construye un nuevo umbral en la producción del conocimiento general y, específicamente, en lo educativo.

¿Qué es el conocimiento post-disciplinar? Si bien es cierto, lo post-disciplinar, a diferencia de lo interdisciplinar, no posee grandes desarrollos analíticos, incluso, sus aplicaciones al diseño de proyectos de investigación, procesos de formación y arquitectura de las estructuras académicas, expresan un halo de baja intensidad - por no decir nula- en el mundo hispanoparlante, no así, en países nórdicos o de habla inglesa. La post-disciplinariedad no presenta grandes desarrollos académicos, por lo que su fuerza conceptualizadora es aún débil. Sus mayores aplicaciones se encuentran vinculadas a campos de la comunicación, el diseño, las artes, no a las Ciencias de la Educación y mucho menos, al dominio de lo educación inclusiva. Ciertamente, las formas definicionales de los prefijos multi-, inter-, para-, alter-, post- y anti-, son confusos, muchas veces se superponen entre sí deviniendo en una práctica de contaminación y confusión entre sus contornos definitorios, y usándose de manera inconsciente. Tal como se ha indicado anteriormente, la post-disciplina orienta su campo de actividad a la producción de lo nuevo que emerge de diversas clases de síntesis heurísticas produciendo algo totalmente nuevo, no aborda problemas de naturaleza disciplinar contribuyendo a interrogar la funcionalidad de las estructuras académicas y sus mecanismos de organización, lo que genera un singular diálogo experimental entre cada uno de sus cuerpos de herramientas y aparatos analítico-metodológicos. Es una forma de escuchar, enunciar, producir e interactuar más allá de cada uno de sus recursos constructivos a través de singulares formas de traducción que contribuirán a reducir las brechas cognitivo-comprehensivas derivadas de la presencia de diversas clases de lenguajes, categorías y jergas especializadas. Surge, entonces, la pregunta acerca de las reglas de funcionamiento del territorio desde una perspectiva post-disciplinar, con el objeto de identificar nuevas preguntas e ideas alterativas de la realidad. La post-disciplina contribuye a investigar problemas específicos de carácter flexible, bajo nuevos proyectos; es sinónimo de enfoques complejos en la producción del conocimiento lo que proporciona nuevos ángulos de visión. La inclusión como campo y fenómeno es un sistema complejo en sí mismo.

El trabajo heurístico desde un punto de vista post-disciplinar exige dislocar las metáforas, pues reconoce que son estas, tensiones restrictivas en la emergencia de nuevas perspectivas, ángulos de visión y herramientas de trabajo. Las metáforas trazan puntos de análisis específico, educan la mirada, el lenguaje y las posiciones del investigador, actúan trazando el norte de las personas y sus concepcio- 
nes acerca de determinados fenómenos. Si no son dislocadas las metáforas recurrentemente empleadas para significar diversas clases de problemas, es altamente probable que la investigación se nutra de suposiciones incorporadas o creencias que conduzcan a agudizar sus errores cognitivos. La producción del conocimiento de la educación inclusiva a través de su estructura falsificada y mixta se mueve a través de creencias preformadas que deforman la comprensión del campo y de sus objetos.

La post-disciplina es una forma de articular posiciones metodológicas, políticas, éticas filosóficas, etc., en torno a objetos móviles, nómades y diaspóricos. No se limita a ningún campo, regionalización intelectual, circunscripción metodológica o paradigmas de ninguna disciplina en particular, etc.; inaugura una forma de indisciplinamiento heurístico. Las post-disciplinas son altamente fértiles en terrenos heurísticos que operan por sistemas transrelacionales y por deslimitación de sus formas, integrados por problemas complejos, poliédricos y con muchas facetas, donde los límites de sus recursos constructivos son plásticos, permeables exigen traducciones, así como, comprensión acerca de sus posiciones y trama de ubicaciones. Lo post-disciplinar brinda mayor posibilidad de movimiento y creatividad, fomenta nuevos mecanismos de ramificaciones, mutaciones, transformaciones; le es fácil entrar y salir de diversos recursos y regionalizaciones; otorga mayores líneas de fuga para abordar una diversidad de problemas.

La educación inclusiva se convierte en un enfoque prometedor en la medida que estructura su campo desde múltiples ensamblajes temáticos. La naturaleza post-disciplinar del campo plantea significativos retos a nivel de producción del conocimiento y del funcionamiento de las estructuras académicas y las prácticas de investigación. La construcción del conocimiento de la educación inclusiva acontece a través de campos, enfoques, geografías epistémicas, recursos metodológicos, instrumentos conceptuales otros, que escapan a la exclusividad de la tiranía de la reflexión filosófica o del análisis sociológico, dos formas comunes al momento de producir entendimientos teóricos en materia de lo educativo. Aquí, nos enfrentamos a una convergencia mucho más compleja: la acción extra-disciplinar. Regresemos a este punto, por unos instantes. Si la educación inclusiva explicita una trayectoria rizomática entonces, esto marca una nueva tendencia y un singular sistema de agenciamiento heurístico-constructivo, que al funcionar en permanente movimiento establece un patrón de dinamismos encarnado, articulando un saber en permanente movimiento, algo provisional cercano a la noción de emergencia temporal. Un territorio post-disciplinar se ensambla a través de un conjunto de prácticas de conocimiento por rearticulación y traducción. Sin duda, esta es la causa mórfica de ensamblaje del campo de producción de lo inclusivo, no como una crisis en sí misma, o falta de fundamentos claros. La educación inclusiva comparte muchos de sus problemas con sus principales campos de confluencia, como algunos de sus enredos genealógicos más trascendentales. Cada uno de estos puntos de contacto que se vuelven legibles mediante singulares tácticas de giro y redoblamiento deslizándose mediante líneas específicas de trabajo, inaugurando un diagrama de posiciones contingentes, situadas y transitivas, por un período determinado. Estas intersecciones emergentes adoptan la forma de aperturas conscientes hacia rumbos desconocidos, forjan una relación sostenible, la que nunca es pacífica y armónica, sino todo lo contrario, se encuentra atenta a los puntos de diálogo entre sus problemas compartidos que se mueven más allá de sus formas comprehensivas conocidas, comprendiendo mejor las complejas interconexiones, transposiciones y traducciones.

La post-disciplinariedad, en tanto actividad heurístico-constructiva, es una respuesta a la descomposición y crisis de la organización tradicional de los cuerpos disciplinarios, desde esta óptica la educación inclusiva introduce nuevos géneros, formatos y apartaos analítico-metodológicos para investigar. Un aspecto clave que se debe relevar es que los aspectos epistemológicos del dominio no adquieren estatus de pre-disciplinar, sino de metáfora. Esta distinción es crucial. Sin embargo, hace bastantes años, sus territorios han adoptado un carácter post-disciplinar, tomando prestados muchos conceptos de otras ramas de conocimientos -injerto y aplicación-, necesita de criterios de legibilidad y posicionalidad de sus funciones lingüístico-analíticas en la gran red móvil de relaciones de lo inclusivo. Las post-disciplinas actúan mediante síntesis creativas de aperturas, poseen la capacidad de subvertir la hegemonía a través del imperialismo conceptual y metodológico. Los territorios de lo inclusivo no son entendidos en términos de totalidad cerrada, sino, de una compleja totalidad dinámica en apertura por redoblamiento 
continuo, gracias a esto, ninguno de sus fenómenos adopta una perspectiva determinista. Algunos de sus fenómenos son de carácter ideológico, político y cultural, no exclusivamente analíticos. La educación inclusiva y la inclusión son sistemas de amplificación de la realidad. Metodológicamente, asumen una descripción acerca del

[...] concepto de 'métodos desordenados'. El enfoque de Law consiste en analizar una variedad de formas diferentes en las que se puede producir conocimiento a través de la investigación y señala que, en realidad, la 'pureza' que asumen la mayoría de los métodos simplemente no conduce a desarrollar una visión representativa de cómo es realmente el mundo. Más bien, el mundo es un lugar desordenado, donde las cosas a menudo no funcionan como deberían. Deben concederse concesiones a la complejidad del mundo; habitualmente tratamos de colocar "elementos cuadrados en agujeros redondos". (p.7)

La educación inclusiva, al producir nuevos objetos de estudio para leer el presente e intervenir en él, requiere de nuevas metodologías. Si bien los contornos del campo son porosos, difusos e indeterminados, estos, a su vez, son propiedades centrales de fenómenos emergentes; forjan un diagrama multiposicional de conocimientos situados y en fuga. La naturaleza del conocimiento de la educación inclusiva reafirma su forma post-disciplinar, micropolítica, diaspórica y nomadista; su carácter de resistencia se funda en acciones encarnadas-realizativas. Heurísticamente, rompe los límites disciplinarios para escuchar y enunciar su campo de problemas más allá de sus formas instituidas. Es una táctica oblicua y de producción que sugiere que las estructuras organizativas de las disciplinas mismas no se mantendrán. La educación inclusiva forja un terreno nuevo, más amplio y transgresor.

La imagen que se expone a continuación sintetiza de forma globalizada la evolución de las diversas conceptualizaciones comúnmente empleadas por la comunidad académica e investigativa para significar el sentido de la diversidad de bases epistemológicas presentes en diversos campos. La imagen ilustra en su último nivel el carácter científico de lo post-disciplinar, que, sea dicho de paso, constituye la base epistemológica de la educación inclusiva.

\section{Figura 1}

Disciplinarities: intra, cross, multi, inter, trans, post

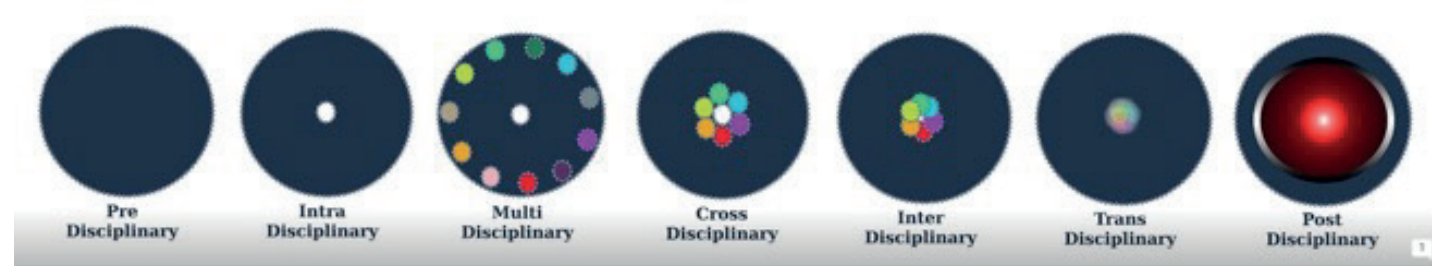

Fuente: Refsum Jensenius (2016)

Finalmente, una interrogante crítica es: ¿qué investiga la educación inclusiva? El giro que ha experimentado progresivamente la fuerza del significante de sus territorios ha disipado, con cierto impacto, los consensos acerca de sus sistemas de filiación y vincularidad con la regionalización de la educación especial y con la fuerza del régimen especial-céntrico que contribuía a imponer un conjunto de suposiciones individualistas-esencialistas, binaristas y universalizadoras acerca de su función. Si bien el campo de relación y los objetos específicos de trabajo de lo inclusivo casi en toda su evolución intelectual ha pseudo-afirmado que no es lo mismo que lo especial; sin embargo, producto de la ambigüedad y debilidad de sus argumentos, recurre a la imposición del modelo epistémico y didáctico de la educación especial, inaugurando una singular modalidad 
de edipización -atrapamiento heurístico, turbiedad cognitiva o sentimiento analítico-metodológico de incomprensión o resentimiento- devenida en figuración denominada 'neo-especial'. El estudio adecuado de la educación inclusiva ya no la forma soberana de lo especial, ni mucho menos, la exclusividad ontológica ligada al sujeto de la alteridad peyorativa, sino, a la trama de relaciones que ensamblan la figura de la pluralidad individual o las múltiples singularidades. La suposición clásica del sujeto de la inclusión - estrechamente ligado a la región del humanismo clásico- desborda las figuras instituidas que nunca han sido neutras, ingenuas y ajenas a singulares mecanismos de poder. Para Braidotti (2018) las concepciones de ser humano históricamente legitimadas entrañan un sistema de "categorización normativa que indexa el acceso a privilegios y derechos" (p.6).

\section{4.}

\section{Conclusiones: tensiones ontológicas y lectura del presente}

La pregunta por las formas definicionales del sujeto social, político y educativo en el marco de la inclusión y la educación inclusiva constituye un punto espinoso; especialmente, por la raigambre de sujeción a la matriz esencialista-individualista sobre la que se fundan los planteamientos onto-céntrico y onto-normativos que son célebremente empleados para justificar la tarea de lo inclusivo a partir de la imposición del legado epistémico y didáctico de la educación especial, ensamblaje que deviene en una singular modalidad de edipización heurística sostenida y reproducida por las agendas de investigación mainstream a través de la figuración de lo neo-especial. Los entendimientos sobre el sujeto educativo tienden a actuar a través de un efecto normativo que refuerza un compromiso con las bases del humanismo clásico y el sustancialismo. En superación de ello, se observa necesario subvertir los códigos de legibilidad de los sujetos educativos y sociales a través de la propuesta del anti-/post-humanismo, que a juicio de Braidotti (2018) no define una nueva condición humana con claridad, pero, fomenta un análisis pormenorizado acerca de la complejidad de procesos -ideológicos, discursivos y relaciones de poder- implicados en la formación del ser.
A la luz de estos argumentos es posible sostener que la educación inclusiva es altamente productiva en su comprensión desde los planteamientos del post-humanismo.

Lo post-humano opera en términos de una cartografía de lectura del presente, sugiere la construcción colectiva de nuevos sujetos de conocimiento y modos de relacionamientos a través de alianzas y nuevas formas pasionales y vinculares con otros actores. La demanda ontológica que asume la educación inclusiva auténtica consiste en construir otros modos de praxis y entendimientos garantes de una nueva humanidad y una compresión-otra del ser humano. Para ello, será necesaria la construcción de una pragmática epistemológica de orden micropolítica dedicada a crear elementos para liberar a los sujetos inscritos en la zona del no-ser o del no-existente, sujetos afectados transrelacionalmente por diversos factores de desigualdad que actúan de forma multiposicional creando una red de problemas estructurales y micro-prácticos. Necesitamos superar las comprensiones de la educación inclusiva, que ficcionalizan la realidad y contribuyen a extender el status quo, mediante la creación de un corpus de códigos de ordenación y acciones que tienden a reconocer los problemas del Otro, sin crear coordenadas de alteridad reales que permitan liberar la vida de las personas. A tal efecto, es necesario consolidar una pragmática epistemológica en materia de educación inclusiva, un saber que penetre la realidad y que transforme la vida de las personas.

La educación inclusiva auténtica o real debe asumir una concepción ontológica y ética próxima a la noción de materialismo encarnado o materialismo nómada, para ello, es fundamental que cree su propia concepción acerca del presente. Una acción crítico-afirmativa con el presente sugiere que

[...] todas las entidades humanas y no humanas son sujetos nómadas en proceso, en perpetuo movimiento, inmanentes a la vitalidad de la materia auto-ordenada. Acercarse al presente produce pues un efecto multifacético: por un lado, la aguda conciencia de lo que somos dejando de ser (el fin de lo actual) y por otro la percepción -en diferentes grados de claridad- de lo que estamos en el proceso de convertirnos (la actualización de lo virtual). 
Ambos fenómenos ocurren a la vez, de forma no lineal, tiempo-continuo. (Braidotti, 2018, p.6-7)

Si bien, la educación inclusiva forja un saber en permanente movimiento y construye un saber del presente, no busca detenerse en la crítica de este, sino que inscribe su fuerza de producción en la actualización creativa de lo virtual, rescata la proposición de Zemelman (1992) acerca de lo dado-dándose-por-dar, aquello que estamos en proceso de llegar a ser, un efecto del materialismo cognoscitivo. Insiste Braidotti (2018), agregando que,

[...] la interacción entre el presente como actual y el presente como virtual deletrea los ritmos de formación del sujeto. Por extensión, lo posthumano como figuración cartográfica es una rama de pensamiento crítico contemporáneo que nos permite pensar en lo que "somos" dejando de ser, por ejemplo, la categoría eurocéntrica del "Hombre" universal. También sostiene, sin embargo, el esfuerzo por dar cuenta de lo que "somos" en el proceso de devenir. (p.7)

La política ontológica de la educación inclusiva se funda en los planteamientos de la revolución molecular, configura un diagrama de posiciones contingentes, relacionales y transitivas que develan que la naturaleza ontológica de la educación inclusiva emerge desde la multitud de formas del ser humano - principio de variabilidad-, nos invita a explorar las formas aún no realizadas del ser; premisa que entraña una sólida empresa creativa. La compresión situada y alterativa de las múltiples formas de lo humano es lo que funda la concepción ontológica de lo menor encarnado en el sintagma onto-epistémico 'singularidades múltiples'. Esta figura ontológica demanda la creación de conceptos para leer un presente caótico, trastornado e impredecible. La historicidad del presente que forja la epistemología de la educación inclusiva puntualiza en

[...] la creatividad, la imaginación, se reconecta constantemente con la totalidad virtual de un bloque de experiencias pasadas y afectos, que se recomponen como acción en el presente, dándose cuenta de su potencial insatisfecho. Este modo de crítica afirmativa es un ejercicio de sincronización temporal y contingente, que sostiene, en el presente, la actividad de actualizar lo virtual. (Braidotti, 2018, p.8)

Si el ser humano se encuentra en proceso de mutación es necesario consolidar una nueva imagen de pensamiento que obligue a reconocer que toda praxis cultural se encuentra incorporada, incrustada por múltiples devenires, por múltiples fuerzas de singularización. La educación inclusiva no cumple con su función ante los retos del mundo contemporáneo, en parte, porque carece de conceptos adecuados para leer el presente. Uno de sus propósitos onto-político clave es aprender a comprender las formas de irrupción de la multiplicidad y sus configuraciones en la vida actual, operación que sugiere organizar las estructuras sociales, políticas y de escolarización en esta dirección.

La educación inclusiva construye un emergente campo de investigación centrado en una diversidad de problemas, no en formas canónicas disciplinantes, concentrándose en una acción post-disciplinar - tal como fue argumentado en secciones anteriores de este trabajo- atravesado por diversas descendencias y trayectorias analítico-metodológicas y convergencias que oscilan entre lo multi-, inter-, trans-, extra- e incluso, anti-disciplinar. Es un territorio que se nutre de aportes que se mueven entre lo instituido - lo canónico- y lo instituyente -campos alejados, ajenos, muchas veces híbridos, emergentes y marginales-, se propone responder a los otros múltiples excedentes del humanismo clásico a través de análisis epistemológicos radicales -algunos de ellos, con más figuración de proyectos políticos-, formas que comparten algunas premisas analítico-metodológicas, tales como, la búsqueda de otros aparatos comprehensivos del ser, la superación de los mecanismos de mono-educacionismo respectos de los problemas de la injusticia, la opresión y la producción de otras formas de desigualdades. La educación inclusiva es un territorio inacabado, compuesto de prácticas de producción del conocimiento en devenir, cuyos problemas medulares se encuentran arraigados en el presente, y cuyo éxodo no solo involucra las pseudo-cadenas hereditarias que definen su ámbito de vinculación con lo especial, sino que traza un multi-éxodo que escapa a las formas definitorias de múltiples pro- 
yectos de conocimiento que participan en su ensamblaje; es una práctica crítica que corre el marco a través de una operación dialéctica anti-hegeliana donde se inscriben las fuerzas de desvinculación y rearticulación.

La naturaleza del conocimiento de la educación inclusiva de carácter post-disciplinar no solo abre nuevos ángulos de visión e interviene en problemas de naturaleza contingente, más bien, afecta a diversos territorios disciplinarios e interdisciplinarios, así como prácticas interpretativas y discursivas -de allí que se sostenga que este es un campo heurístico que actualiza a las disciplinas-, lo que explícita una "nueva energía metadiscursiva por parte de las disciplinas" (Braidotti, 2018, p.13). El carácter post-disciplinario de la educación inclusiva no solo reafirma que su fenómeno no pude ser delimitado en los paradigmas de ninguna disciplina en particular, sino que establece una red de agenciamientos heurísticos que proceden mediante diversas formas y aparatos de descendencia híbridas y ensamblajes heterogéneos, consolidando relaciones epistémicas sin precedentes. La educación inclusiva presenta, además, una naturaleza porosa y un centro epistémico relacional; territorio complejo, dinámico, que parece nunca detenerse. El carácter post-disciplinar de la educación inclusiva es un ensamblaje de diversas clases de recursos supradisciplinares comprometidos con las demandas del devenir minoritario, en adelante, mayorías en el mundo. Impone la tarea de develar el orden heurístico de aceleración de lo menor. Es esto lo que impulsa a modos alterativos de comprender al ser y a sus tramas de devenir.

\section{Referencias}

Bal, M. (2009). Conceptos viajeros en las humanidades. Una guía de viaje. Murcia: Cendeac.

Biagioli, M. (2009). Postdisciplinary Liaisons: Science Studies and the Humanities. Critical Inquiry, 35(4). https://doi.org/10.1086/599586
Brah, A. (2011). Cartografías de la diáspora. Identidades en cuestión. Madrid: Traficantes de Sueños.

Braidotti, R. (2018). A Theoretical Framework for the Critical Posthumanities. Theory, Culture \& Society, 36(6), 31-61. https://doi.org/10.1177/0263276418771486

De Landa, M. (2016). Assemblage Theory. Edimburgo: Edinburgh University Press.

Martínez, M. (3 de julio de 2020). Félix Guattari: de la Revolución molecular a la Ecosofía (II). El salto diario. https://bit.ly/2ZJcfX2

Mengue, Ph. (2008). Deleuze o el Sistema de lo múltiple. Buenos Aires: los cuarenta libros.

Niinimäki, K., Groth, C., Kääriäinen, P. (2018). NEW SILK: Studying experimental touchpoints between material science, synthetic biology, design and art. Temes de Disseny, (34), 34-43, https://doi.org/10.46467/TdD34.2018.34-43

Ocampo, A. (2017). Epistemología de la educación inclusiva. Granada: UGR.

Panayotov, S. (11 de septiembre de 2016). Diagram. New Materialism. How matter comes to matter. https://bit.ly/3qSAUUX

Refsum, A. (2016). Disciplinarities: intra, cross, multi, inter, trans, post. https://www.arj.no/2012/03/12/disciplinarities-2/

Sousa, B. (2009). Una epistemología del sur: la reinvención del conocimiento y la emancipación social. México: Siglo XXI/Clacso.

Zemelman, H. (1992). Los horizontes de la razón. I. Dialéctica y apropiación del presente. México: Antrophos. 
